\section{ACTAS Dermo-Sifiliográficas}

Full English text available at www.actasdermo.org

\title{
Documento de consenso español para el algoritmo de tratamiento de la rosácea
}

\author{
M. Salleras ${ }^{a, *}$, M. Alegre ${ }^{b}$, V. Alonso-Usero ${ }^{c}$, P. Boixeda ${ }^{d}$, J. Domínguez-Silva ${ }^{e}$, \\ J. Fernández-Herrera ${ }^{f}$, X. García-Navarro ${ }^{g}, N$. Jiménez ${ }^{d}, M$. Llamas ${ }^{f}$, C. Nadal ${ }^{\text {h }}$, \\ J. del Pozo-Losada ${ }^{i}$, I. Querol ${ }^{j}$, I. Salguiero ${ }^{k}, M$. Schaller ${ }^{\prime}$ y J. Soto de Delás ${ }^{m}$
}

\author{
a Servicio de Dermatología, Hospital Universitario Sagrado Corazón, Barcelona, España \\ b Servicio de Dermatología, Hospital Plató, Barcelona, España \\ c Servicio de Dermatología, Hospital Nisa 9 de Octubre, Valencia, España \\ d Sección de Dermatología, Hospital Ramón y Cajal, Madrid, España \\ e Servicio de Dermatología, Hospital Universitario de Gran Canaria Doctor Negrín, Las Palmas de Gran Canaria, España \\ f Sección de Dermatología, Hospital de La Princesa, Madrid, España \\ s Servicio de Dermatología, Consorci Sanitari del Garraf, Barcelona, España \\ h Servicio de Dermatología, Hospital Son Llatzer, Palma de Mallorca, España \\ i Servicio de Dermatología, Hospital Universitario A Coruña, La Coruña, España \\ j Universidad de Zaragoza, Zaragoza, España \\ k Servicio de Dermatología, Hospital Universitario Puerta de Hierro Majadahonda, Madrid, España \\ ' Departamento de Dermatología, Universidad de Klinikum, Tubinga, Alemania \\ m Servicio de Dermatología, Policlínica Gipuzkoa, San Sebastián, España
}

Recibido el 3 de agosto de 2018; aceptado el 1 de enero de 2019

\section{PALABRAS CLAVE \\ Rosácea; \\ Brimonidina; \\ Metronidazol; \\ Doxiciclina; \\ Fuentes de luz; \\ Isotretinoína; \\ Ivermectina}

\begin{abstract}
Resumen Las últimas evidencias científicas y la incorporación de nuevos fármacos al arsenal terapéutico de la rosácea hacen necesario revisar y actualizar los criterios y estrategias de tratamiento. Con este fin, un grupo de 15 dermatólogos expertos en esta enfermedad aportaron y discutieron acerca de las diferentes terapias y los criterios de respuesta y cambio de tratamiento. Partiendo de la revisión crítica de la bibliografía y de la exposición de los hábitos de los dermatólogos españoles en su práctica clínica, se formularon distintas propuestas que fueron debatidas teniendo en consideración tanto la experiencia profesional como las preferencias de los pacientes o los criterios de equidad. Una vez validadas las propuestas, se formularon las recomendaciones finales que, junto con la evidencia aportada por las principales guías y estudios internacionales, dieron lugar al presente documento. El objetivo de este consenso es ofrecer al dermatólogo un enfoque práctico para abordar la rosácea.

(c) 2019 AEDV. Publicado por Elsevier España, S.L.U. Todos los derechos reservados.
\end{abstract}

\footnotetext{
* Autor para correspondencia.

Correo electrónico: msalleras@quironsalud.es (M. Salleras).
} 


\section{KEYWORDS}

Rosacea;

Brimonidine;

Metronidazole;

Doxycycline;

Light sources;

Isotretinoin;

Ivermectin

\section{Spanish Consensus Document on the Treatment Algorithm for Rosacea}

Abstract Recent scientific evidence and the incorporation of new drugs into the therapeutic arsenal against rosacea have made it necessary to review and update treatment criteria and strategies. To this end, a panel of 15 dermatologists, all experts in rosacea, was formed to share experiences and discuss treatment options, response criteria, and changes to treatment. Based on a critical review of the literature and a discussion of the routine practices of Spanish dermatologists, the panel proposed and debated different options, with consideration of the experience of professionals and the preferences of patients or equality criteria. Following validation of the proposals, the final recommendations were formulated and, together with the evidence from the main international guidelines and studies, used to produce this consensus document. The goal of this consensus document is to provide dermatologists with practical recommendations for the management of rosacea.

(C) 2019 AEDV. Published by Elsevier España, S.L.U. All rights reserved.

\section{Introducción}

La rosácea es un trastorno crónico frecuente de la piel que afecta predominantemente al rostro, caracterizado por la aparición de rubor o enrojecimiento, pápulas y pústulas. A menudo, también compromete a los ojos y puede evolucionar a un engrosamiento de la piel con aumento de volumen, denominada fima ${ }^{1}$.

Aunque los datos epidemiológicos son dispares, estudios realizados en Europa y Estados Unidos estiman una incidencia entre menos del $1 \%$ y más del $20 \%$ de la población general $^{2}$. No obstante, la comparativa es difícil, ya que difiere notablemente tanto la metodología empleada como la población estudiada en cada caso. Como ejemplo, algunos trabajos determinan una prevalencia global del $2,2 \%$ en Alemania $^{3}$, de entre el 1,3 y el 2,1\% en Estados Unidos ${ }^{4}$ o del $22 \%$ en Estonia ${ }^{5}$.

La aparición de la enfermedad suele producirse entre los 30 y los 50 años $^{6}$, con mayor frecuencia en mujeres que en hombres ${ }^{4}$, aunque estos son más proclives al desarrollo de fimas $^{7}$. Si bien la rosácea puede afectar a todos los grupos raciales y étnicos, parece que las personas de piel clara son más propensas ${ }^{6}$.

En cuanto a su patogenia, son pocos los estudios realizados; sin embargo, algunos datos apoyan la hipótesis de la predisposición genética como clave en la enfermedad ${ }^{8,9}$, con una alteración en la inmunidad innata. También se ha demostrado la existencia de factores predisponentes y desencadenantes, como ciertos factores ambientales ${ }^{2}$, alimentarios, emocionales o el uso de algunos productos tópicos ${ }^{10}$.

A pesar de la evidencia científica, el manejo de la rosácea adolece en ocasiones de desigualdad de criterio en lo referente a su diagnóstico y tratamiento, no siempre basados en los diferentes subtipos clínicos ${ }^{4}$. Por otra parte, en los últimos años se han producido muchos avances en el tratamiento del eritema facial y de las lesiones inflamatorias ${ }^{11,12}$, por lo que se ha hecho imperativo revisar y actualizar los principales criterios y estrategias terapéuticas.
Para elaborar este documento se han incluido guías y revisiones sobre rosácea, entre ellos la última actualización del consenso de la American Acne and Rosacea Society, así como la revisión Cochrare Interventions for rosacea y el consenso alemán Rosacea-S1 guideline ${ }^{13-18}$. Así, el presente documento de consenso recoge las últimas recomendaciones basadas en la mejor evidencia disponible ${ }^{19}$, incluyendo la evaluación de la enfermedad, los objetivos de tratamiento o el esquema terapéutico. Pretende servir de referencia a los dermatólogos implicados en el tratamiento de la rosácea y de guía práctica en su toma de decisiones. Quedan excluidos de la intención de este documento el abordaje de formas más infrecuentes y complejas de la enfermedad, como la demodicosis, la rosácea granulomatosa, la rosácea fulminans, etc. Tampoco se abordará ni revisará la idoneidad de los complementos cosméticos habitualmente empleados para dicha afección (limpiadores, emolientes, fotoprotectores...) que, si bien fueron reconocidos como indispensables por el panel de expertos, no fueron objeto de discusión.

\section{Metodología}

Como grupo de expertos, se formó un panel de 15 dermatólogos seleccionados en función de su nivel de conocimientos, experiencia clínica e investigadora (ver currículum en material suplementario) De ellos, uno actuó como coordinador (MS), formulando y presentando las propuestas, y el resto las discutieron y validaron.

El coordinador enunció el índice temático de preguntas de relevancia clínica que fue la base para la elaboración de las recomendaciones. El coordinador recopiló e interpretó los datos disponibles tras la revisión bibliográfica y elaboró el listado de recomendaciones que sería sometido a consenso por el grupo de dermatólogos participantes.

Con el fin de conocer el abordaje de la rosácea por parte del panel de expertos en su práctica clínica habitual, se circuló un cuestionario con 23 preguntas articuladas en función de su interés con relación al diagnóstico y 
Tabla 1 Cuestionario inicial para conocer el perfil de los expertos y su abordaje de la rosácea

Para rellenar por los expertos

1. Filiación del dermatólogo

Indique su edad:

$<30$

$31-40$

41-50

Más de 50

Provincia donde ejerce:

Lugar donde ejerce su actividad profesional:

Ámbito privado

Seguridad social

Ambos

2. ¿De cuál de los dos ámbitos proviene la mayoría de su consulta?

Ámbito privado

Seguridad social

3. Aproximadamente, ¿cuántos pacientes acuden a su consulta de media a la semana?

Menos de 30

Entre 30-50

Más de 50

4. Aproximadamente, ¿cuántos pacientes acuden a su consulta por problemas de rosácea, en una semana normal?

0-5 por semana

5-10 por semana

10-15 por semana

$>15$ por semana

5. ¿Está de acuerdo con la clasificación de la rosácea en eritematotelangiectásica, papulopustulosa, fimatosa y ocular?

Sí (en este caso, ¿utiliza esta clasificación en su práctica clínica?)

No (en este caso, especifique la clasificación que utiliza en su práctica clínica)

6. ¿Qué criterio sigue para determinar el grado de rosácea del paciente?

Subtipo clínico anterior

Número de lesiones (en la papulopustulosa)

Impacto sobre la calidad de vida (DLQI)

7. En el caso de rosácea subtipo 1 o eritematotelangiectásica, ¿cuál es su modo de proceder?

Solo la trato a petición del paciente (DLQI)

Sugiero al paciente un tratamiento si la evidencio clínicamente

La trato siempre, pues la considero un factor de riesgo para evolucionar a rosácea papulopustulosa

No la trato

8. En el caso de rosácea subtipo 2 o papulopustulosa:

¿Hasta qué número de lesiones considera que está ante una rosácea leve?

0-10 lesiones

11-20 lesiones

$>20$ lesiones

¿Hasta qué número de lesiones considera que está ante una rosácea moderada?

0-10 lesiones

11-20 lesiones

$>20$ lesiones

¿A partir de qué número de lesiones considera que está ante una rosácea severa?

0-10 lesiones

11-20 lesiones

$>20$ lesiones

La aparición de nódulos/granulomas, ¿modifica su percepción de gravedad de la rosácea?

Sí, la considero directamente como severa

Sí, la considero severa a partir de 2 nódulos

Sí, la considero severa a partir de 3 nódulos

No, no modifica mi percepción de gravedad de la rosácea

9. ¿Cómo se distribuyen los pacientes con rosácea que acuden a su consulta en función de su gravedad?

Rosácea leve

Rosácea moderada

Rosácea grave 
Tabla 1 (continuación)

10. ¿Es importante para usted el problema de las resistencias antibióticas?

Sí

No

11. En su opinión, ¿los tratamientos dermatológicos contribuyen a las resistencias antibióticas?

Sí

No

12. ¿Considera usted el problema de las resistencias antibióticas al elegir su estrategia de tratamiento para la rosácea? Sí

No

13. ¿Qué tratamiento prescribe más frecuentemente para el tratamiento de la rosácea? (principio activo)

Tópicos: metronidazol

Ácido azelaico

Ivermectina

Clindamicina

Eritromicina

Sulfacetamida

Otros

Orales: minociclina

Doxiciclina a dosis subantimicrobiana: $40 \mathrm{mg}$

Doxiciclina a dosis ATB: 50/100/200 mg

Metronidazol

Macrólidos (eritromicina, claritromicina, azitromicina)

Otros

14. De media, ¿por cuánto tiempo prescribe la terapia antibiótica tópica en pacientes con rosácea?

$0-1,5$ meses

1,5-3 meses

3 meses -2 meses

Indefinidamente

15. De media, ¿por cuánto tiempo prescribe un antibiótico oral (dosis antimicrobiana) en pacientes con rosácea?

$0-1,5$ meses

1,5-3 meses

3 meses- 12 meses

Indefinidamente

16. ¿Qué efecto busca cuando prescribe un antibiótico (oral o tópico) para el tratamiento de la rosácea?

Antiinflamatorio

Antibacteriano

Ambos

17. ¿Sobre cuál de las siguientes afirmaciones se basa para prescribir antibióticos tópicos en la rosácea?

Es indispensable para tratar una enfermedad donde un ácaro contribuye a la patología

Prescribo antibióticos tópicos solo por su efecto antiinflamatorio

No me siento cómodo no usándolos para tratar una enfermedad que es infecciosa

El paciente pide un tratamiento antibiótico

Por hábito

18. ¿Sobre cuál de las siguientes afirmaciones se basa para prescribir antibióticos orales en la rosácea?

Es indispensable para tratar una enfermedad donde un ácaro contribuye a la patología

Prescribo antibióticos orales solo por su efecto antiinflamatorio

No me siento cómodo no usándolos para tratar una enfermedad que es infecciosa

El paciente pide un tratamiento antibiótico

Por hábito

La dosis subantimicrobiana de doxiciclina $(40 \mathrm{mg})$ es efectiva para tratar las lesiones papulopustulosas de la rosácea, sin generar resistencia bacteriana

19. ¿Cuál es la razón principal por la que prescribe una isotretinoína oral?

Ante el fracaso de los antibióticos orales

No quiero usar antibióticos orales porque no es una enfermedad infecciosa

Gravedad de la rosácea

A petición del paciente 
Tabla 1 (continuación)

20. ¿Considera usted los siguientes factores cuando prescribe una isotretinoína oral? (marque todas las aplicables)

Efectos secundarios/intolerancia cutánea propia de la rosácea

Tratamientos previos fallidos

Disposición del paciente

Necesidad de incluir un tratamiento anticonceptivo femenino

Efectos suspensivos y no curativos de la isotretinoína

Otros (indique cuáles)

21. ¿Cuál es su estrategia de tratamiento considerando el tipo de lesión?(en caso de poner 2 o más opciones se entenderá que las utiliza de forma combinada

Primera línea

Segunda línea

Mantenimiento

22. ¿Utiliza cosméticos en asociación a los tratamientos convencionales

(limpiadores/fotoprotectores/emolientes/maquillajes)?

Sí (siempre/en la mayoría de los casos/solo en mujeres)

No

Si la respuesta es sí, ¿cuál es su pauta de utilización?

Los utilizo previamente al tratamiento con un medicamento

Los utilizo al final del tratamiento

Los utilizo al inicio y al final del tratamiento

23. ¿Cree que el tratamiento de la rosácea es completo en la actualidad?

Sí

No. ¿Qué lagunas terapéuticas quedan por cubrir?

DLQI: Dermatology Quality of Life Index.

tratamiento de la enfermedad (tabla 1). Los resultados de las preguntas más relevantes del cuestionario completado por el panel de expertos se muestran en la tabla S1 como material suplementario. Con el resultado de la encuesta y de la revisión bibliográfica, el coordinador redactó las recomendaciones que serían tratadas mediante consenso, principalmente sobre aquellas cuestiones en las que se observó diversidad de opinión o falta de evidencia.

\section{Búsqueda y selección bibliográfica}

Para la selección de artículos, se realizó una búsqueda bibliográfica en las siguientes bases de datos: Medline/Pubmed, The Cochrane Library y Google Scholar. La búsqueda se centró en revisiones y artículos originales en inglés o español publicados entre los años 2002 y 2015. Los filtros metodológicos definidos para acotar la búsqueda fueron revisiones, series de casos, estudios de prevalencia, estudios de cohortes, casos y controles, estudios no aleatorizados, ensayos clínicos controlados aleatorizados y metaanálisis que incluían a pacientes con rosácea. Se realizó una selección de aquellos estudios que ofrecían datos más útiles para responder a las preguntas clínicas, asegurándose de que las recomendaciones estuvieran basadas en la mejor evidencia. Para evaluar la calidad y validez de los estudios se emplearon las plantillas del CASPe (Critical Appraisal Skills Programme Español ${ }^{20}$, cuyos resultados definieron el nivel de evidencia en cada caso y determinaron su correspondiente impacto en la recomendación.

A continuación, se estimó la calidad de la evidencia de todos resultados y se calificó la fuerza de las recomendaciones mediante un enfoque sistemático y explícito utilizando el sistema de clasificación GRADE (Grading of
Recommendations Assessment, Development and

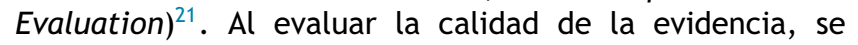
incluyó información sobre los defectos metodológicos de los estudios, la consistencia de los resultados, su probable generalización y la magnitud del efecto. Una vez revisada y evaluada la bibliografía, se elaboraron las correspondientes recomendaciones con base en la mejor evidencia posible, experiencia clínica y aplicabilidad. Tras su redacción, se clasificaron y priorizaron para su implementación en la ronda de preguntas, en la que serían tratadas mediante discusión por el panel de expertos.

\section{Procedimiento de elaboración del Consenso}

Se plantearon 20 enunciados agrupados en 4 áreas temáticas: a) definición de rosácea, b) repercusión en la calidad de vida, c) enfoque terapéutico, d) tratamiento. Para su validación, el grupo de trabajo celebró una reunión presencial en la que se discutieron de forma abierta todos los planteamientos. Los expertos debatieron individualmente las recomendaciones, propusieron mejoras y redactaron las recomendaciones finales.

Para la medición del consenso se utilizó la escala Likert ${ }^{22}$, por la que se consideró consenso cuando al menos el $75 \%$ de los expertos estuvo de acuerdo o totalmente de acuerdo con lo planteado. En una primera ronda de votaciones se consensuaron 17 recomendaciones, 8 de ellas con un alto grado de consenso. Las 2 restantes fueron validadas en una segunda ronda. En total, fueron aprobadas 19 recomendaciones y 4 algoritmos de tratamiento, uno por cada subtipo de rosácea (fig. S1, material suplementario). La tabla 2 resume las recomendaciones emitidas, con base en el consenso de los expertos o en la evidencia disponible. 
Tabla 2 Lista de las recomendaciones emitidas por el panel de expertos

Recomendación

Base de la recomendación

La rosácea es una enfermedad cutánea crónica frecuente, de sintomatología variable, que afecta generalmente a la región centrofacial

La clasificación de la rosácea en 4 subtipos resulta práctica para tipificar los resultados de la investigación clínica

Los subtipos clínicos de la rosácea son útiles para la clasificación de signos y síntomas; sin embargo, no aclaran aspectos como la cronicidad o la evolución de las características individuales de la enfermedad

A nivel psicosocial, la rosácea puede afectar negativamente el bienestar del individuo. A la hora de plantear el abordaje terapéutico, los dermatólogos debemos tener en cuenta su impacto en la calidad de vida del paciente

Es conveniente respetar el tiempo de respuesta al tratamiento, siempre que haya tolerancia a este, antes de cambiar a otra opción alternativa

Ante la falta de respuesta a un tratamiento de primera línea, se puede emplear otro fármaco establecido de primera línea o añadir un segundo agente terapéutico

Cuando se elija una terapia de mantenimiento, debe individualizarse cada caso y se aconseja elegir la terapia mínima eficaz que logre el control de los síntomas

Ante la presencia de signos/síntomas severos de rosácea, puede optarse por emplear más de una terapia simultáneamente

Puede considerarse el uso de brimonidina tópica o betabloqueantes orales para el tratamiento del eritema transitorio o flushing

Para el eritema no transitorio, el láser de colorante pulsado y la luz pulsada intensa son tratamientos de primera elección; brimonidina puede emplearse como tratamiento sintomático

IPL y láser vasculares son tratamientos de primera línea para las telangiectasias

Para la rosácea con lesiones inflamatorias (pápulas, pústulas) leves, los agentes tópicos como ivermectina y metronidazol se recomiendan como primera línea. Puede considerarse doxiciclina oral como una alternativa

Para la rosácea con lesiones inflamatorias (pápulas, pústulas) moderadas, los agentes tópicos como ivermectina o metronidazol o doxiciclina oral son tratamientos de primera línea

Para la rosácea con lesiones inflamatorias (pápulas, pústulas) graves, se recomienda tratamiento sistémico (doxiciclina o isotretinoína) asociado o no a terapia tópica (ivermectina o metronidazol) o física como primera línea de tratamiento

Cuando se decida el empleo de tetraciclinas para tratar las lesiones inflamatorias de la rosácea, se recomienda el uso de doxiciclina a la dosis mínima eficaz a fin de minimizar la aparición de resistencias bacterianas

Para lesiones fimatosas activas, se recomienda el uso de tratamiento sistémico como primera línea (doxiciclina o isotretinoína)

Para lesiones fimatosas sin inflamación clínica, se recomienda la realización de procedimientos quirúrgicos como la dermoabrasión, electrodesecación o láser. Por su capacidad de esculpir y hacer hemostasia simultáneamente y de forma controlada es preferible el láser $\mathrm{CO}_{2}$ escaneado o pulsado, en modo continuo

Los dermatólogos deben reconocer las manifestaciones oculares de la rosácea. Se debe derivar al oftalmólogo a aquellos pacientes que presenten alteraciones visuales, afectación conjuntival o indicios de afectación corneal

El tratamiento de la rosácea ocular depende del grado de afectación. Comprende medidas generales como la limpieza adecuada, terapias tópicas (metronidazol, tetraciclinas o ciclosporina) y terapia sistémica (doxiciclina, pautas cortas de corticoides o isotretinoína)
Consenso de

expertos

Consenso de expertos

Consenso de

expertos

Basada en la evidencia $^{25}$

Consenso de expertos

Consenso de

expertos

Consenso de expertos

Consenso de expertos

Basada en la evidencia $^{11,26}+$ Consenso de expertos Basada en la evidencia $^{18}$

Consenso de expertos

Basada en la evidencia $^{32,33}$

Basada en la evidencia $^{15,18,34}$

Basada en la evidencia $^{16,19}$

Basada en la evidencia $^{17,18,35}$

Consenso de expertos

Consenso de expertos

Consenso de expertos

Consenso de expertos
NA NA

Calidad de la evidencia/fuerza de recomendación

NA

NA

NA

Calidad moderada Recomendación fuerte

NA

NA

NA

NA

Calidad alta Recomendación fuerte

NA

Calidad alta Recomendación fuerte

NA

Calidad moderada Recomendación fuerte

Calidad alta Recomendación fuerte Calidad alta Recomendación fuerte

Calidad alta Recomendación fuerte NA

NA

IPL: luz intensa pulsada.

Cómo citar este artículo: Salleras M, et al. Documento de consenso español para el algoritmo de tratamiento de la rosácea. Actas Dermosifiliogr. 2019. https://doi.org/10.1016/j.ad.2019.01.001 


\section{Resultados del Consenso para el algoritmo de tratamiento de la rosácea}

\section{Definición de rosácea}

La rosácea es una enfermedad cutánea crónica común que afecta principalmente a las convexidades centrofaciales (mejillas, mentón, nariz y centro de la frente). Se trata de una condición cutánea que incluye diferentes signos como flushing, eritema, telangiectasias, edema, pápulas, pústulas, lesiones oculares y fimas. En la mayoría de los casos aparecen solo algunos de estos signos, pero en otros los pacientes pueden presentar simultáneamente más de uno ${ }^{23}$.

A pesar de la vigente clasificación por subtipos (subtipo 1 o rosácea eritematotelangiectásica, subtipo 2 o rosácea papulopustulosa, subtipo 3 o rosácea ocular y subtipo 4 o rosácea fimatosa), todavía existe escasez de información sobre los signos y síntomas de la rosácea, la relación entre los subtipos y el potencial de progresión entre ellos ${ }^{24}$.

En opinión del grupo de expertos, la clasificación de la rosácea en 4 subtipos no convence clínicamente a los especialistas, pero es práctica desde el punto de vista de la investigación.

Recomendaciones emitidas:

La rosácea es una enfermedad cutánea crónica frecuente, de sintomatología variable, que afecta generalmente a la región centrofacial.

La clasificación de la rosácea en 4 subtipos resulta práctica para tipificar los resultados de la investigación clínica.

Los subtipos clínicos de la rosácea son útiles para la clasificación de signos y síntomas; sin embargo, no aclaran aspectos como la cronicidad o la evolución de las características individuales de la enfermedad.

\section{Repercusión en la calidad de vida}

La rosácea afecta principalmente a la cara, y su repercusión en la calidad de vida del paciente es indiscutible. Todos los estudios de calidad de vida en rosácea han evidenciado que los pacientes pueden experimentar sentimientos de vergüenza y ansiedad ${ }^{25}$. Asimismo, pueden presentar falta de confianza, baja autoestima, una imagen corporal negativa y depresión. Comprender la experiencia del paciente con relación a la calidad de vida es importantísimo para el correcto manejo de la enfermedad o la elección del tratamiento adecuado. Igualmente, conocer las comorbilidades (migraña, trastornos gastrointestinales...) que puedan asociar condicionará la elección del agente terapéutico.

Al utilizar un tratamiento, es importante tener en cuenta cuál es el tiempo necesario para lograr una respuesta de cada principio activo, así como su duración. También es fundamental prestar atención a los efectos adversos que produce cada medicamento ${ }^{1}$.

El perfil psicológico del paciente con rosácea en algunos casos es el de un paciente ansioso debido a experiencias previas de fracasos terapéuticos o resultados insuficientes. Si la respuesta es lenta, se debe insistir el tiempo suficiente para que el medicamento pueda mostrar su eficacia, excepto en el caso de que el paciente no lo tolere bien. En práctica clínica habitual, los tratamientos del brote suelen alargarse 6-8 semanas.
Si bien, en general, se dice que cuando el tratamiento de primera línea no es suficiente se pasa al de segunda línea, para la rosácea, hay varios tratamientos de primera línea, y fue difícil encontrar una escala de preferencias en cuanto a los principios activos tópicos entre los participantes en el consenso.

Recomendaciones emitidas:

En el ámbito psicosocial, la rosácea puede afectar negativamente el bienestar del individuo. A la hora de plantear el abordaje terapéutico, los dermatólogos debemos tener en cuenta su impacto en la calidad de vida del paciente.

Es conveniente respetar el tiempo de respuesta al tratamiento, siempre que haya tolerancia, antes de cambiar a otra opción alternativa.

Ante la falta de respuesta a un tratamiento de primera línea, se puede emplear otro fármaco establecido de primera línea o añadir un segundo agente terapéutico.

\section{Enfoque terapéutico:}

El tratamiento de la rosácea debe basarse en los subtipos y, según este diagnóstico, se escogerá el o los principios activos adecuados. Los consejos generales para los pacientes con rosácea incluyen medidas de higiene suaves, uso frecuente de emolientes y uso habitual de fotoprotectores con índices de protección altos (SPF 30-50). Estas medidas generales de tratamiento, así como la eliminación o atenuación de los factores agravantes de la rosácea (climáticos, dietéticos, etc.), son muy importantes para el manejo de la sintomatología habitualmente presente en esta condición cutánea, como la sequedad, el prurito y la sensación de quemazón, y fueron reconocidos por los expertos presentes en el consenso, si bien no fueron evaluados.

¿Qué ocurre con el tratamiento de mantenimiento? ¿Es necesario hacerlo siempre o no? ¿Solamente si el paciente lo quiere? La naturaleza crónica de la rosácea justifica el uso de un tratamiento de mantenimiento, que dependerá de la evolución clínica del paciente. Si bien algunos entran en remisión y permanecen así durante mucho tiempo, otros presentan frecuentes recaídas a muy corto plazo. Con un tratamiento de mantenimiento, el paciente puede permanecer entre 1 y 3 años sin presentar lesiones, dependiendo del grado de la enfermedad. Es necesario individualizar el tratamiento de mantenimiento. Deberían tenerse en cuenta el criterio y la recomendación del médico, aunque la decisión final de realizarlo recae sobre el paciente. El propósito de administrar un tratamiento de mantenimiento es disminuir la probabilidad de recidiva y, generalmente, consiste en un tratamiento tópico, aunque la decisión final habrá que acordarla con el paciente y tener en cuenta sus preferencias.

En el tratamiento de la rosácea moderada o grave, el uso combinado de terapia tópica y oral es el abordaje óptimo, aunque se basará en la gravedad según el dermatólogo y el paciente (grado de los síntomas y el deseo del paciente de obtener resultados más o menos rápidamente ${ }^{17}$ ).

Recomendaciones emitidas:

Cuando se elija una terapia de mantenimiento, se debe individualizar cada caso y se aconseja elegir la terapia mínima eficaz que logre el control de los síntomas. 
Ante la presencia de signos o síntomas severos de rosácea, puede optarse por emplear más de una terapia simultáneamente.

\section{Tratamiento}

Eritema/telangiectasias: subtipo I de rosácea

El eritema facial, tanto el basal (eritema no transitorio o permanente) como el que aparece temporalmente sin aparente motivo o bien inducido por los factores desencadenantes (eritema transitorio o flushing), es probablemente el aspecto más común de la rosácea y una de las manifestaciones que más preocupa a los pacientes.

El tratamiento tópico puede ser suficiente para controlar el eritema (los agonistas alfa-2 adrenérgicos tópicos inducen la vasoconstricción de los vasos cutáneos superficiales, reduciendo el eritema de fondo durante varias horas después de la aplicación), pero no actúa en las telangiectasias ${ }^{17}$. Los láseres y fuentes de luz como la luz pulsada intensa pueden utilizarse también para tratar el eritema y son la opción principal para el tratamiento de las telangiectasias ${ }^{17,18}$.

Eritema transitorio (flushing). El tartrato de brimonidina tópico en gel al 0,5\% administrado una vez al día, por la mañana, ha sido evaluado en 2 estudios fase III aleatorizados, doble-ciego, controlados con vehículo en el tratamiento del eritema moderado-severo permanente y transitorio (flushing) de la rosácea ${ }^{11,26}$. Estos estudios demuestran su eficacia en alrededor de un $70 \%$ de casos. Su efecto se inicia a los 30 min de la aplicación, es máximo hacia las $3 \mathrm{~h}$, se mantiene hasta las $9 \mathrm{~h}$ y posteriormente decrece hasta la desaparición total del efecto hacia las $12 \mathrm{~h}$ postaplicación. La brimonidina, por tanto, está indicada en el tratamiento del enrojecimiento facial causado por la rosácea ${ }^{27,28}$ y es un tratamiento de primera línea frente a este síntoma. Aunque constituye el avance más significativo en el tratamiento del eritema transitorio en la rosácea en algunos pacientes, sin embargo, es mal tolerado. Además, conviene puntualizar que es un tratamiento sintomático, no definitivo, tal y como viene especificado en su ficha técnica ${ }^{29}$.

El láser y las fuentes de luz, de forma aislada o en combinación con brimonidina, siguen constituyendo uno de los pilares fundamentales en el tratamiento del eritema ${ }^{27,30}$, aunque estos tratamientos físicos son más efectivos en las telangiectasias y mucho menos en el eritema, sobre todo el inducido por desencadenantes, en el que generalmente solo producen mejoría parcial.

El flushing se puede tratar también con oximetazolina tópica al $1 \%$, y oralmente con clonidina, betabloqueantes (propranolol, carvedilol), naloxona y ondansetrón. No obstante, la evidencia que apoya algunas de estas terapias es limitada ${ }^{28}$. El grupo de expertos apoya la utilización de los betabloqueantes orales y otros antihipertensivos como segunda opción de tratamiento (off label) del eritema transitorio o flushing.

Eritema no transitorio o permanente. El eritema facial no transitorio o permanente en la rosácea se puede tratar con láseres específicos como el láser de colorante pulsado (siglas en inglés, PDL) y fuentes de luz, como la luz intensa pulsada (siglas en inglés, IPL) ${ }^{16}$, que han demostrado una eficacia similar $^{18}$. El uso de brimonidina en monoterapia como tratamiento sintomático del eritema permanente también puede ser una buena opción ${ }^{18}$. El panel de expertos recomienda la combinación PDL o IPL con brimonidina como primera opción de tratamiento.

Como se ha mencionado, para el eritema transitorio algunos fármacos antihipertensivos pueden ser utilizados para el tratamiento del eritema permanente. Los betabloqueantes, que causan vasoconstricción de los vasos sanguíneos arteriales cutáneos mediante el bloqueo del receptor $\beta_{2}$ adrenérgico arterial, se utilizan off label para el tratamiento del eritema ${ }^{31}$. El panel de expertos refirió una experiencia discreta con el empleo de estos fármacos, y aconseja el uso de láser vascular y betabloqueantes orales (off label) como segunda opción de tratamiento del eritema permanente.

Telangiectasias. Tanto el PDL como la IPL resultan beneficiosos para tratar las telangiectasias ${ }^{30}$. Los láseres y fuentes de luz son la primera opción de tratamiento de las telangiectasias y se pueden utilizar de forma combinada.

Existió controversia en el grupo de expertos acerca del uso de la electrodesecación como uno de los posibles tratamientos de las telangiectasias. Generalmente, se considera que el bisturí eléctrico no es tratamiento de primera elección para este tipo de lesiones, aunque a falta de otra tecnología y en manos expertas se podrían obtener buenos resultados. Por lo que el panel de expertos posiciona el uso de electrodesecación como segunda opción de tratamiento de las telangiectasias.

Recomendaciones emitidas:

Se puede considerar el uso de brimonidina tópica o betabloqueantes orales para el tratamiento del eritema transitorio o flushing.

Para el eritema no transitorio, el láser de colorante pulsado y la luz pulsada intensa son tratamientos de primera elección; brimonidina puede emplearse como tratamiento sintomático.

IPL y láser vasculares son tratamientos de primera línea para las telangiectasias.

El algoritmo de tratamiento consensuado para la rosácea eritematotelangiectásica o subtipo I se presenta en la tabla 3.

Rosácea papulopustulosa: subtipo II de rosácea

En general, en la primera línea de tratamiento para las lesiones inflamatorias leves se puede elegir entre utilizar ivermectina o metronidazol tópicos o doxiciclina oral, aunque su eficacia no es exactamente la misma en esta indicación ${ }^{1}$.

Si bien en consensos europeos e internacionales el ácido azelaico se recoge también como opción de primera línea de tratamiento ${ }^{17,18}$, de acuerdo con el panel de expertos, en España, las encuestas indican que su utilización es muy escasa como tratamiento de primera línea. Por el contrario, sí se emplea frecuentemente como tratamiento complementario, o de mantenimiento entre brotes. En consecuencia, la decisión de tratamiento está básicamente entre metronidazol e ivermectina ${ }^{14,32}$. En algunos países como Estados Unidos, también es habitual el empleo de la sulfacetamida asociada a bioazufre, no comercializados en nuestro país.

En la práctica clínica actual metronidazol e ivermectina se emplean según la experiencia del dermatólogo y las características del paciente (adherencia a la pauta posológica, nivel socioeconómico, cobertura sanitaria, 
Tabla 3 Esquema de tratamiento en eritema/telangiectasias

\begin{tabular}{|c|c|c|c|c|}
\hline & & Eritema transitorio & $\begin{array}{l}\text { Eritema no } \\
\text { transitorio }\end{array}$ & Telangiectasias \\
\hline \multirow[t]{2}{*}{$\begin{array}{l}\text { 1. }{ }^{\mathrm{a}} \text { opción de } \\
\text { tratamiento }\end{array}$} & Monoterapia & Brimonidina tópica & $\begin{array}{l}\text { PDL } \\
\text { IPL } \\
\text { Brimonidina } \\
\text { tópica }^{\text {a }}\end{array}$ & $\begin{array}{l}\text { PDL } \\
\text { IPL }\end{array}$ \\
\hline & Combinación & $\begin{array}{l}\text { Brimonidina tópica } \\
\text { + láser vascular o } \\
\text { IPL }\end{array}$ & $\begin{array}{l}\text { PDL o IPL } \\
\text { +Brimonidina } \\
\text { tópica }\end{array}$ & $P D L+I P L$ \\
\hline \multirow[t]{2}{*}{$\begin{array}{l}\text { 2. }{ }^{a} \text { opción de } \\
\text { tratamiento }\end{array}$} & Monoterapia & $\begin{array}{l}\text { Betabloqueantes } \\
\text { orales (off label) }\end{array}$ & $\begin{array}{l}\text { Otros láseres } \\
\text { vasculares } \\
\text { Betabloqueantes } \\
\text { orales (off label) }\end{array}$ & Electrodesecación \\
\hline & Combinación & & & \\
\hline
\end{tabular}

IPL: intense pulsed light; PDL: pulsed dye laser.

a Tratamiento sintomático.

etc.). Cabe destacar que un estudio fase III aleatorizado y ciego para el investigador, con 960 pacientes, demostró superioridad de la crema de ivermectina $1 \%$ una vez al día frente a metronidazol $0,75 \% 2$ veces al día en la semana 16 del tratamiento $(83,0$ vs. $73,3 \% ; p<0,001)$ con tendencia a mostrar un aumento de la eficacia incluso en pacientes con mayor gravedad. Ivermectina además mostró mejor perfil de seguridad y mejor tolerancia que metronidazol ${ }^{32}$.

Por otro lado, Stein Gold et al. llevaron a cabo un estudio en el que se evaluó la terapia tópica a largo plazo en la rosácea papulopustulosa, comparando ivermectina al $1 \%$ en crema con ácido azelaico al $15 \%$ en gel como «tratamientos de mantenimiento». Fueron 2 estudios de extensión a partir de 2 estudios iniciales de fase III de 40 semanas de duración (controlados y ciegos para el investigador). En ellos se demostró que el porcentaje de pacientes «limpios o casi limpios» utilizando la escala IGA fue más elevado al final que en la evaluación inicial del estudio en el grupo que siguió tratamiento con ivermectina, y que la tolerancia y seguridad a lo largo de todo el seguimiento fue también superior para ivermectina que para ácido azelaico ${ }^{33}$.

El tratamiento tópico suele ser suficiente en pacientes con rosácea papulopustulosa leve. Pero en enfermedad moderada-grave, se suele combinar con tratamiento sistémico ${ }^{17,19}$. Ivermectina, metronidazol y ácido azelaico son de nuevo las opciones de primera línea en el tratamiento tópico de las lesiones papulopustulosas y el eritema perilesional de intensidad moderada ${ }^{17}$. Y las tetraciclinas la primera opción de tratamiento oral.

Algunos estudios que han evaluado metronidazol o ácido azelaico en combinación con doxiciclina oral han demostrado que la combinación agente tópico más agente oral es más eficaz y más rápida que el uso de los tópicos en monoterapia, sin un incremento de efectos adversos ${ }^{14}$. Asimismo, estudios de fase III han demostrado que doxiciclina ( $40 \mathrm{mg} /$ día, dosis antiinflamatoria, no antibiótica) puede usarse como primera línea de tratamiento en monoterapia para rosácea papulopustulosa ${ }^{34} \mathrm{y}$, de hecho, las guías americana y alemana ya la recomiendan para el subtipo II de rosácea ${ }^{15,18}$.

Del Rosso et al. compararon la seguridad y la eficacia de la dosis antiinflamatoria de doxiciclina $(40 \mathrm{mg}$, de liberación retardada una vez al día) con la dosis antibiótica de $100 \mathrm{mg}$ / día durante 16 semanas en un estudio multicéntrico, prospectivo, aleatorizado, doble-ciego y controlado $(N=96)$. Los autores concluyeron que ambas dosis eran igualmente eficaces en el tratamiento de la rosácea grave, aunque la dosis de $40 \mathrm{mg}$ presentaba ventajas respecto a la dosis antibiótica, debido al menor riesgo de efectos adversos y al hecho de que no contribuye al desarrollo de resistencias bacterianas $^{35}$. Asimismo, el consenso americano destaca que $40 \mathrm{mg}$ doxiciclina evitan la resistencia antibiótica ${ }^{17}$.

Controversia sobre el uso de antibióticos en rosácea. La controversia sobre el uso de dosis antimicrobianas de tetraciclinas (50-200 mg) frente al uso de dosis solo antiinflamatorias ( $40 \mathrm{mg}$ ) fue ampliamente discutida en la reunión de consenso.

Las terapias antiinflamatorias se consideran las más apropiadas para el manejo de la rosácea, especialmente para la rosácea papulopustulosa, ya que la rosácea se considera una enfermedad inflamatoria y no infecciosa. Sin embargo, el grupo de expertos reconoció que en práctica clínica habitual se siguen usando antibióticos para controlar esta afección. El tratamiento antibiótico oral más utilizado es la dosis antibiótica de doxiciclina (50-200 mg/día), que se indica en los casos que responden mal a una terapia tópica razonable. Solo a veces se indica una dosis subantimicrobiana de doxiciclina $(40 \mathrm{mg} / \mathrm{día})^{17}$. Minociclina a dosis de $100 \mathrm{mg} /$ día es otra tetraciclina muy empleada que ha demostrado eficacia en el tratamiento de la rosácea papulopustulosa ${ }^{18}$, así como otras tetraciclinas en los países en las que están disponibles.

En pacientes con alergia, intolerancia o resistencia a tetraciclinas, o bien ante contraindicaciones como el embarazo o edad menor de 10 años, se recurre a los macrólidos como eritromicina, claritromicina y azitromicina. Eritromicina oral a una dosis de 250-1.000 mg diarios se considera un tratamiento muy eficaz, aunque debido a sus efectos secundarios no se suele recomendar ${ }^{18}$ y se prefiere el uso de otros macrólidos.

Así, aunque los consensos internacionales recalcan que no es conveniente utilizar dosis antimicrobianas de antibióticos para el tratamiento de la rosácea ${ }^{17,18}$ ya que con la administración de estos fármacos, en realidad, se busca solo el efecto antiinflamatorio y este efecto se obtiene ya con 
dosis de tan solo $40 \mathrm{mg}$ de doxiciclina, el grupo de expertos insistió en que según su experiencia personal, tiene una mayor eficacia la dosis de $100 \mathrm{mg}$ y que probablemente, lo ideal sería indicar una dosis alta de tetraciclinas de inicio y una dosis baja de mantenimiento de $40 \mathrm{mg} /$ día. Con dicha dosis, se producirían menos efectos secundarios (como candidiasis, menos resistencias, etc.). Esta forma de actuar se reconoció como la más recomendable, aunque no siempre coincide con la realidad en la práctica clínica.

Las rosáceas graves o que no responden al tratamiento estándar se pueden tratar con isotretinoína oral. La efectividad de isotretinoína oral para el tratamiento de la rosácea grave está demostrada ${ }^{19}$. Cuando se trate de rosácea papulopustulosa refractaria al tratamiento en la que se excluya la baja adherencia, isotretinoína oral puede ser una opción en casos cuidadosamente seleccionados ${ }^{17}$ y a dosis más bajas que las empleadas en el acné $(0,25 \mathrm{mg} / \mathrm{kg}$ al día), aunque a diferencia de lo que ocurre con el acné, el tratamiento de la rosácea con isotretinoína supone un tratamiento suspensivo no «curativo».

Finalmente, los sistemas láser como PDL y láser de neodimio-YAG, así como las fuentes de luz tipo IPL se pueden utilizar también en estas rosáceas catalogadas como graves para tratar de forma efectiva los vasos cutáneos superficiales dilatados de manera persistente que no responden a las terapias médicas empleadas en el tratamiento de las papulopústulas, incluyendo las telangiectasias lineales y las redes telangiectásicas más confluentes ${ }^{16}$.

Recomendaciones emitidas:

Para la rosácea con lesiones inflamatorias (pápulas, pústulas) leves, los agentes tópicos como ivermectina y metronidazol se recomiendan como primera línea. Se puede considerar doxiciclina oral como una alternativa.
Para la rosácea con lesiones inflamatorias (pápulas, pústulas) moderadas, los agentes tópicos como ivermectina o metronidazol o doxiciclina oral son tratamientos de primera línea.

Para la rosácea con lesiones inflamatorias (pápulas, pústulas) graves, se recomienda tratamiento sistémico (doxiciclina o isotretinoína) asociado o no a terapia tópica (ivermectina o metronidazol) o física como primera línea de tratamiento.

Cuando se decida el empleo de tetraciclinas para tratar las lesiones inflamatorias de la rosácea, se recomienda el uso de doxiciclina a la dosis mínima eficaz a fin de minimizar la aparición de resistencias bacterianas.

El algoritmo de tratamiento consensuado para la rosácea papulopustulosa o subtipo II se muestra en la tabla 4.

Rosácea fimatosa: subtipo III de rosácea

Lesiones fimatosas activas (con inflamación). Tanto doxiciclina como isotretinoína oral han demostrado eficacia en el tratamiento de las lesiones fimatosas activas ${ }^{15,19}$. Isotretinoína se emplea a dosis bajas $(0,1-0,5 \mathrm{mg} / \mathrm{kg}$ al día) durante 6-8 meses $^{19,36}$. Este fármaco ha demostrado tener el potencial de retrasar la progresión de las fimas ${ }^{37}$. Según algunos autores, cuando coexisten varios subtipos de rosácea, suele ser beneficioso combinar láser vascular y doxiciclina o isotretinoína oral. También IPL ha demostrado buenos resultados en varios escenarios clínicos ${ }^{30}$.

El panel de expertos recomienda la combinación de láser vascular o IPL con doxiciclina o isotretinoína oral (off label) en los pacientes con lesiones fimatosas activas. Como segunda opción de tratamiento, el panel de expertos recomienda la administración de otros antibióticos orales o antiparasitarios orales (metronidazol u otros).

Tabla 4 Esquema de tratamiento en rosácea papulopustulosa

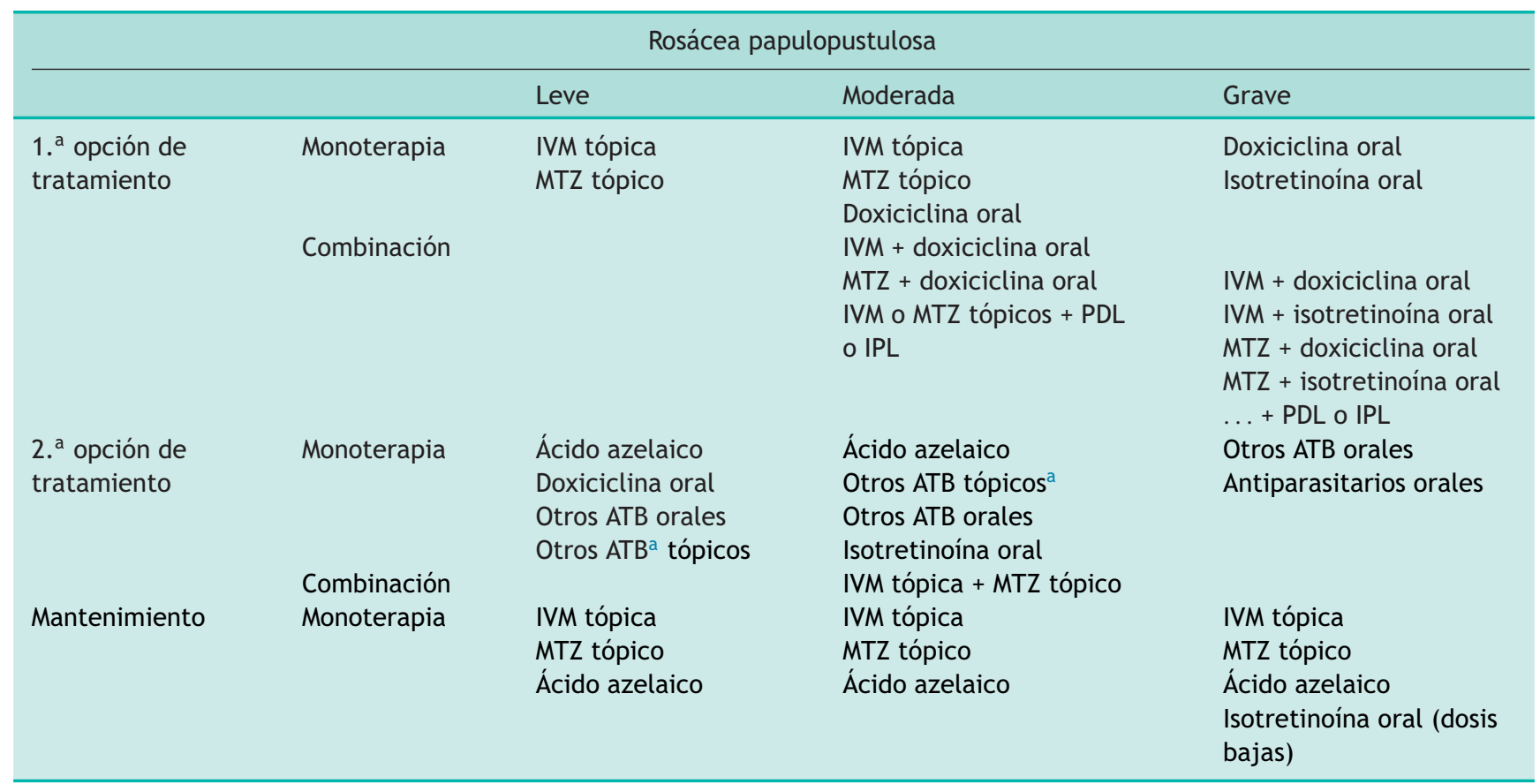

ATB: antibiótico; IPL: intense pulsed light; IVM: ivermectina; MTZ: metronidazol; PDL: pulsed dye laser.

a Sulfacetamida, eritromicina, clindamicina. 
Es conveniente administrar terapia de mantenimiento para evitar la reaparición de las lesiones. El grupo de especialistas aconseja utilizar fármacos tópicos como ivermectina, metronidazol o ácido azelaico.

Lesiones fimatosas estables o no activas. De acuerdo con la evidencia científica, en el tratamiento de las fimas se deben utilizar terapias físicas, bien sea láser o tratamiento quirúrgico ${ }^{16,18}$. Debido a que no existe ninguna terapia médica efectiva para el tratamiento definitivo de la rosácea con cambios fimatosos completamente desarrollados, las modalidades físicas o las intervenciones quirúrgicas se utilizan habitualmente con varios manejos posibles. Estos abordajes, que algunas veces consisten en una combinación de terapias, incluyen la escisión tangencial y dermoabrasión. Se ha eliminado el electrobisturí, la ablación por láser, las tijeras para esculpir y la electrocirugía ${ }^{16,18}$.

Recomendaciones emitidas:

Para lesiones fimatosas activas, se recomienda el uso de tratamiento sistémico como primera línea (doxiciclina o isotretinoína).

Para lesiones fimatosas sin inflamación clínica, se recomienda la realización de procedimientos quirúrgicos como la dermoabrasión, electrodesecación o láser. Por su capacidad de esculpir y hacer hemostasia simultáneamente y de forma controlada, se prefiere el láser $\mathrm{CO}_{2}$ escaneado o pulsado, en modo continuo.

El algoritmo de tratamiento consensuado para la rosácea fimatosa o subtipo III se presenta en la tabla 5 .

Afectación ocular: subtipo IV de rosácea

Existe afectación ocular en el 30-50\% de los pacientes con rosácea cutánea. Para el tratamiento de las formas leves puede ser suficiente el uso de tratamiento local oftalmológico. La higiene del borde del párpado es una opción, así como el uso de lágrimas artificiales con base lipídica ${ }^{18}$.

Algunos fármacos han demostrado eficacia en la reducción de la inflamación local en este tipo de rosácea, como corticoides tópicos, metronidazol al 0,75\% y ciclosporina tópica al 0,05\% 2 veces al día ${ }^{18}$. En aquellos casos en que los síntomas oculares persisten, doxiciclina (50-100 mg, 2 veces al día) o bien doxiciclina a dosis antiinflamatoria $(40 \mathrm{mg}$ al día) han mostrado buenos resultados ${ }^{15,18}$. Asimismo, el tratamiento con dosis bajas de isotretinoína, $10 \mathrm{mg} /$ día, produce mejoría en los síntomas oculares ${ }^{19}$.

En opinión del panel de expertos, en líneas generales, ni ciclosporina ni pautas cortas de corticoides se utilizan con frecuencia en el tratamiento de la rosácea en España. Los especialistas prefieren metronidazol y tetraciclinas tópicas $y$, en cuanto a los tratamientos orales, tetraciclinas.

La opinión de los dermatólogos con relación al tratamiento de la rosácea ocular puede entrar en conflicto con la de los oftalmólogos. Una buena opción sería realizar reuniones conjuntas dermatólogo-oftalmólogo para tratar dicha afección. Actualmente los pacientes con rosácea ocular leve son tratados por dermatólogos y los casos moderado-graves son derivados al oftalmólogo.

Recomendaciones emitidas:

Los dermatólogos deben reconocer las manifestaciones oculares de la rosácea. Se debe derivar al oftalmólogo a aquellos pacientes que presenten alteraciones visuales, afectación conjuntival o indicios de afectación corneal.

El tratamiento de la rosácea ocular depende del grado de afectación. Comprende medidas generales como la limpieza adecuada, terapias tópicas (metronidazol, tetraciclinas o ciclosporina) y terapia sistémica (doxiciclina, pautas cortas de corticoides o isotretinoína).

El algoritmo de tratamiento consensuado para la rosácea ocular o subtipo IV se describe en la tabla 6.

Tabla 5 Esquema de tratamiento en rosácea fimatosa

\begin{tabular}{|c|c|c|c|}
\hline \multicolumn{4}{|c|}{ Rosácea fimatosa } \\
\hline & & Activa & Estable \\
\hline \multirow[t]{2}{*}{$\begin{array}{l}\text { 1. }{ }^{\mathrm{a}} \text { opción de } \\
\text { tratamiento }\end{array}$} & Monoterapia & $\begin{array}{l}\text { Doxiciclina } \\
\text { orlsotretinoína oral }\end{array}$ & $\begin{array}{l}\text { Láser } \mathrm{CO}_{2} \text { escaneado o } \\
\text { pulsado } \\
\text { Dermoabrasión } \\
\text { Electrocirugía } \\
\text { Cirugía }\end{array}$ \\
\hline & Combinación & $\begin{array}{l}\text { Doxiciclina oral + PDL o } \\
\text { IPL } \\
\text { Isotretinoína oral (Off } \\
\text { label) + láser vascular o } \\
\text { IPL }\end{array}$ & $\begin{array}{l}\text { Combinación de terapias } \\
\text { físicas }\end{array}$ \\
\hline $\begin{array}{l}\text { 2. }{ }^{\mathrm{a}} \text { opción de } \\
\text { tratamiento }\end{array}$ & Monoterapia & $\begin{array}{l}\text { Otros ATB orales } \\
\text { Antiparasitarios orales }\end{array}$ & \\
\hline Mantenimiento & $\begin{array}{l}\text { Combinación } \\
\text { Monoterapia }\end{array}$ & $\begin{array}{l}\text { Metronidazol tópico } \\
\text { Ivermectina tópica } \\
\text { Ácido azelaico }\end{array}$ & \\
\hline & Combinación & & \\
\hline
\end{tabular}

ATB: antibiótico; IPL: intense pulsed light; PDL: pulsed dye laser. 
Tabla 6 Esquema de tratamiento en afectación ocular

\begin{tabular}{lll}
\hline & Afectación ocular & \\
\hline 1a opción de tratamiento & Monoterapia & -Metronidazol tópico \\
& & -Tetraciclina tópica \\
& & -Doxiciclina oral \\
& Combinación & -Metronidazol tópico + Doxiciclina oral \\
$2 \underline{a}$ opción de tratamiento & Monoterapia & -Ciclosporina tópica \\
Mantenimiento & Combinación & -Isotretinoína oral \\
& Monoterapia & -Metronidazol tópico \\
\hline
\end{tabular}

Los pacientes con afectación ocular deben ser derivados al oftalmólogo para su valoración.

\section{Limitaciones del trabajo}

En general, en la aplicación de la metodología Delphi para generar consensos debe tenerse en cuenta el potencial sesgo de los participantes derivado de su experiencia y ámbito asistencial, que puede afectar a la hora de dar su opinión sobre diferentes situaciones clínicas. Sin embargo, la opinión subjetiva de un grupo de especialistas siempre será de mayor calidad que la opinión de un solo especialista, debido a la mayor cantidad de información de la que dispone un grupo. La búsqueda bibliográfica efectuada para recabar la evidencia disponible no fue sistemática, sino dirigida a las preguntas planteadas por el coordinador. Además, hay que tener en cuenta que los artículos publicados a partir del 2016 no se han incluido como parte de la bibliografía, por lo que no se descarta que nuevos estudios puedan modificar algunos puntos del algoritmo terapéutico de la rosácea.

\section{Conclusiones}

El tratamiento de la rosácea se basa tanto en la gravedad de sus signos y síntomas como en su presentación clínica que, en muchos casos, consiste en una combinación de varias características de los diferentes subtipos de esta entidad. Junto con las medidas generales de limpieza e hidratación de la piel, habitualmente es preciso ofrecer al paciente una combinación de terapias farmacológicas, tal como se describe en el presente documento. En la actualidad existe una mayor sensibilización en los especialistas en relación con el impacto de la rosácea en la calidad de vida, lo que conlleva que el dermatólogo implique al paciente en las decisiones acerca de su tratamiento.

\section{Financiación}

Galderma ha colaborado en la financiación de la reunión presencial de trabajo del grupo de expertos. El laboratorio financiador no ha participado en la redacción del artículo.

\section{Conflicto de intereses}

Monserrat Salleras ha colaborado con Galderma como coordinadora y participante en la reunión para el consenso de tratamiento de la rosácea, como ponente en el lanzamiento de brimonidina, como ponente local en charlas de actualización de rosácea para el equipo de dermatólogos del Hospital Universitario Sagrat-Cor y ha recibido ayudas para desplazamiento/inscripción a congresos de dermatología. Para la elaboración de este artículo, declara no tener conflicto de intereses con relación a los tratamientos propuestos, los cuales ha defendido exclusivamente con relación a su experiencia clínica. Marta Alegre ha participado como ponente de Galderma en 2 ocasiones y en 2 advisory boards de Galderma. Los autores Vicent Alonso Usero, Pablo Boixeda y Jesús Domínguez Silva expresan que no existen conflictos de intereses con Galderma. Jesús Fernández Herrera ha participado como ponente de Galderma; declara no tener otros conflictos de interés. No ha participado en ensayos clínicos ni recibido ayudas para desplazamiento/inscripción a congresos ni becas para la realización de proyectos científicos. Xavier García Navarro declara no tener conflicto de interés con Galderma. Natalia Jiménez declara haber participado en 2 advisory boards con Galderma. Mar Llamas ha participado como ponente de Galderma en una ocasión. Con relación a los tratamientos empleados en la rosácea discutidos en el consenso, no tiene otros conflictos de interés que declarar. No ha participado en ensayos clínicos con Galderma ni recibido ayudas para desplazamiento/inscripción a congresos ni becas para la realización de proyectos científicos. Cristina Nadal ha participado como ponente en una reunión de Galderma y en un advisory board (consenso de rosácea). Jesús del Pozo Losada declara no tener otros conflictos de interés. Ignacio Querol e Irene Salgüero expresan que no existen conflictos de interés con Galderma. Martin Schaller ha participado en advisory boards organizados por Bayer, Galderma y Marpinion en los 2 últimos años y ha recibido honorarios como ponente de AbbVie, Bayer Healthcare, Galderma y La Roche-Posay. Jorge Soto de Delás ha participado como ponente con Galderma; declara no haber participado en ensayos clínicos con sus productos, ni haber recibido ayudas para desplazamiento/inscripción a congresos ni becas para la realización de proyectos científicos.

\section{Anexo. Material adicional}

Se puede consultar material adicional a este artículo en su versión electrónica disponible en doi:10.1016/j. ad.2019.01.001. 


\section{Bibliografía}

1. Intervenciones para la rosácea (Revisión Cochrane traducida). Cochrane Database Syst Rev. 2015;4(CD003262). Doi: 10.1002/14651858.CD003262.

2. García-Bustíndy M, García-Fumero V. Rosácea. Impacto psicosocial. Piel (Barc). 2016;31:96-195.

3. Schaefer I, Rustenbach SJ, Zimmer L, Augustin M. Prevalence of skin diseases in a cohort of 48,665 employees in Germany. Dermatology. 2008;217:169-72.

4. Barona MI, Orozco B, Motta A, Meléndez E, Flaminio R, Pabón JG, et al. Rosácea: actualización. Piel (Barc). 2015, https://doi.org/10.1016/j.piel.2015.02.010.

5. Abram K, Silm H, Oona M. Prevalence of rosacea in an Estonian working population using a standard classification. Acta Derm Venereol. 2010;90:269-73.

6. Chosidow O, Cribier B. Epidemiology of rosacea: Updated data. Ann Dermatol Venereol. 2011;138 Suppl 3:S179-83.

7. Powell FC. Clinical practice. Rosacea. N Engl J Med. 2005;352:793-803.

8. Steinhoff M, Buddenkotte J, Aubert J, Sulk M, Novak P, Schwab VD, et al. Clinical, cellular, and molecular aspects in the pathophysiology of rosacea. J Investig Dermatol Symp Proc. 2011;15:2-11.

9. Abram K, Silm H, Maaroos HI, Oona M. Risk factors associated with rosacea. J Eur Acad Dermatol Venereol. 2010;24:565-71.

10. Gil-Díaz MJ, Boixeda de Miguel JP, Truchuelo-Díez M, MoraisCardoso P. Rosácea: revisión y nuevas alternativas terapéuticas. Semergen. 2011;37:83-6.

11. Fowler J Jr, Jackson M, Moore A, Jarratt M, Jones T, Meadows $\mathrm{K}$, et al. Efficacy and safety of once-daily topical brimonidine tartrate gel $0.5 \%$ for the treatment of moderate to severe facial erythema of rosacea: Results of two randomized, doubleblind, and vehicle-controlled pivotal studies. J Drugs Dermatol. 2013;12:650-6.

12. Stein L, Kircik L, Fowler J, Tan J, Draelos Z, Fleischer A, et al. Efficacy and safety of ivermectin $1 \%$ cream in treatment of papulopustular rosacea: Results of two randomized, doubleblind, vehicle-controlled pivotal studies. J Drugs Dermatol. 2014;13:316-23.

13. Del Rosso JQ, Thiboutot D, Gallo R, Webster G, Tanghetti E, Eichenfield L, et al. Consensus recommendations from the American Acne \& Rosacea Society on the management of rosacea, part 1: a status report on the disease state, general measures, and adjunctive skin care. Cutis. 2013;92:234-40.

14. Del Rosso JQ, Thiboutot D, Gallo R, Webster G, Tanghetti E, Eichenfield L, et al. Consensus recommendations from the American Acne \& Rosacea Society on the management of rosacea, part 2: A status report on topical agents. Cutis. 2013;92:277-84.

15. Del Rosso JQ, Thiboutot D, Gallo R, Webster G, Tanghetti E, Eichenfield LF, et al. Consensus recommendations from the American Acne \& Rosacea Society on the management of rosacea, part 3: A status report on systemic therapies. Cutis. 2014;93:18-28.

16. Tanghetti E, Del Rosso JQ, Thiboutot D, Gallo R, Webster G, Eichenfield LF, et al. Consensus recommendations from the American acne \& rosacea society on the management of rosacea, part 4: A status report on physical modalities and devices. Cutis. 2014;93:71-6.

17. Del Rosso JQ, Thiboutot D, Gallo R, Webster G, Tanghetti E, Eichenfield LF, et al. Consensus recommendations from the American Acne \& Rosacea Society on the management of rosacea, part 5: A guide on the management of rosacea. Cutis. 2014;93:134-8.

18. Van Zuuren EJ, Fedorowicz Z, Carter B, van der Linden MM, Charland L. Interventions for rosacea. Cochrane Database Syst Rev. 2015:CD003262, http://dx.doi.org/10. 1002/14651858.CD003262.pub5.
19. Shidan E, Vicaut E, Chidiak H, Anselin E, Cribier B, Dréno B, et al. A randomized controlled trial of oral low dose isotretinoin for difficult to treat papulopustular rosacea. J Invest Dermatol. 2016;136:1124-9.

20. Programa en habilidades de lectura crítica español. España [Internet].CASPE [consultado 12 mayo 2016]. Disponible en: http: //.redcaspe.org.

21. GRADE working group [Internet] [consultado 12 mayo 2016]. Disponible en: http://www.gradeworkinggroup.org/ es/faq/index.htm.

22. Tastle WJ, Wierman M. Consensus and dissention: A measure of ordinal dispersion Internat. J Approx Reason. 2007;45:531-45.

23. Wilkin J, Dahl M, Detmar M, Drake L, Feinstein A, Odom $\mathrm{R}$, et al. Standard classification of rosacea: Report of the National Rosacea Society Expert Committee on the Classification and Staging of Rosacea. J Am Acad Dermatol. 2002;46: 584-7.

24. Tan J, Blume-Peytavi U, Ortonne JP, Wilhelm K, Marticou L, Baltas $E$, et al. An observational cross-sectional survey of rosacea: Clinical associations and progression between subtypes. $\mathrm{Br} \mathrm{J}$ Dermatol. 2013;169:555-62.

25. Van der Linden MM, van Rappard DC, Daams JG, Sprangers MA, Spuls PI, de Korte J. Health-related quality of life in patients with cutaneous rosacea: A systematic review. Acta Derm Venereol. 2015;95:395-400.

26. Fowler J, Jarratt M, Moore A, Meadows K, Pollack A, Steinhoff M, et al. Once-daily topical brimonidine tartrate gel $0.5 \%$ is a novel treatment for moderate to severe facial erythema of rosacea: Results of two multicentre, randomized and vehicle-controlled studies. Br J Dermatol. 2012;166:633-41.

27. National Health Service (NHS). Rosacea-treatment. 2014 [consultado 17 julio 2016]. Disponible en: http://www.nhs.uk/ Conditions/Rosacea/Pages/Treatment.aspx.

28. Culp B, Scheinfeld N. Rosacea: A review. P T. 2009;34:38-45.

29. Ficha técnica Brimonidina. Mirvaso((Laboratorios Galderma S.A.). Disponible en: http://www.ema.europa.eu/docs/ es_ES/document_library/EPAR__Product_Information/human/002642/WC500163121.pdf Último acceso: octubre 2016.

30. Weinkle AP, Doktor V, Emer J. Update on the management of rosacea. Clin Cosmet Investig Dermatol. 2015;8:159-77.

31. Steinhoff M, Schmelz M, Schauber J. Facial erythema of rosacea. Aetiology different pathophysiologies and treatment options. Acta Derm Venereol. 2016;96:579-86.

32. Taieb A, Ortonne JP, Ruzicka T, Roszkiewicz J, Berth-Jones J, Peirone $\mathrm{MH}$, et al. Superiority of ivermectin $1 \%$ cream over metronidazole $0,75 \%$ cream in treating inflammatory lesions of rosacea: A randomized, investigator-blinded trial. $\mathrm{Br} \mathrm{J}$ Dermatol. 2015;172:1103-10.

33. Stein Gold L, Kircik L, Fowler J, Jackson JM, Tan J, Draelos $\mathrm{Z}$, et al. Long-term safety of ivermectin $1 \%$ cream vs azelaic acid $15 \%$ gel in treating inflammatory lesions of rosacea: results of two 40-week controlled, investigator-blinded trials. J Drugs Dermatol. 2014;13:1380-6.

34. Del Rosso JQ, Webster GF, Jackson M, Rendon M, Rich P, Torok $\mathrm{H}$, et al. Two randomized phase III clinical trials evaluating anti-inflammatory dose doxycycline (40-mg doxycycline USP capsules) administered once daily for treatment of rosacea. J Am Acad Dermatol. 2007;56:791-802.

35. Del Rosso JQ, Schlessinger J, Werschler P. Comparison of anti-inflammatory dose doxycycline versus doxycycline $100 \mathrm{mg}$ in the treatment of rosacea. J Drugs Dermatol. 2008;7: $573-6$.

36. Elsaie ML, Choudhary S. Updates on the pathophysiology and management of acne rosacea. Postgrad Med. 2009;121:178-86.

37. Korting HC, Schöllmann C. Current topical and systemic approaches to treatment of rosacea. J Eur Acad Dermatol Venereol. $2009 ; 23: 876-82$. 